P. C. H. Martens, S. Tsuruta, and M. A. Weber, eds.

\title{
Pulsar Geometrodynamics: Relativistic Radiative Plasma Theory and its Associated Quantum Phenomena
}

\author{
A. A. da Costa \\ Centro de Electrodinâmica, Instituto Superior Técnico, 1049-001, \\ Lisboa, Portugal
}

\begin{abstract}
Plasma motion in pulsar magnetospheres is quasi-classical due to curvature radiation of highly energetic gamma-ray photons, implying an extension to the kinetic theory of plasmas. But with high energies involved, other quantum radiative processes become important in the context of vacuum (quantum) electrodynamics.
\end{abstract}

\section{Introduction: Relativistic Plasma Theory}

Pulsar electron-positron magnetospheric plasmas are high energy moving media. This plasma is immersed in a low-frequency, strong electromagnetic (EM) wave with a high value of the magnetic induction $B$. The dominant parameter in such plasmas is the strength of the EM wave given by $\eta=e A_{0} / m c$ (da Costa \& Kahn 1982; da Costa 1983), where $A_{0}$ is the strength of the vector potential. The parameter $\eta \sim 10^{16}$ at the surface of the star for $B \sim 10^{8} \mathrm{~T}$, and $\eta \sim 10^{6}-10^{10}$ at the speed of light cylinder (SLC) for periods $T_{\mathrm{p}} \sim 33 \mathrm{~ms}-3 \mathrm{~s}$.

The parameter $\eta$ is related to the density of the electromagnetic energy $w_{\mathrm{em}} \sim 10^{23} \mathrm{~J} \mathrm{~m}^{-3}$ for $B \sim 10^{8} \mathrm{~T}$, and $w_{\mathrm{em}} \sim 10^{11} \mathrm{~J} \mathrm{~m}^{-3}$ for $B \sim 10^{2} \mathrm{~T}$-its value at the SLC for the Crab pulsar (CP). The Lorentz force and its associated power $P_{\text {Lor }}$ dominates any other interaction in the process, allowing the single particle approach (SPA).

The accelerated plasma reaches very high values of $\gamma$, and starts radiating curvature radiation. The power radiated $P_{\text {rad }}$ grows with $\gamma$, up to $P_{\text {rad }} \sim P_{\text {Lor }}$. The motion is now described by the Lorentz-Dirac equation (L-DEM), to take into account radiative effects (da Costa \& Kahn 1981).

Let us consider a simplified two dimensional model of the pulsar, resembling the field of the equatorial plane of the perpendicular model, where the electric field $E \perp B$ (da Costa \& Kahn 1981), with a plasma starting its motion at the stellar surface and sliding along magnetic field lines (MFL). It is shown that, for the CP and close to the SLC, along the MFL bent in the forward direction of motion, the particles have so much inertia that they leave the MFL. For the CP, particles reach $\gamma \sim 10^{7}$, with a peak frequency $\omega_{0} \sim 10^{23} \mathrm{~Hz}$, i.e. hard gamma-ray radiation (da Costa \& Kahn 1981). Then $P_{\text {Lor }} \lesssim P_{\text {rad }}$. All particles with the same initial conditions will follow the same trajectory.

In the L-DEM description, a classical (continuous) radiation field influences the trajectory of the particle in a continuous way, but the gamma-ray photons radiated by the plasma produce discontinuous effects. The radiative reaction 
correction has to be substituted by a random term, showing radiation of photons at random times, frequency and direction-a Markovian process. This is the quasi-classical (q-classical) regime (da Costa \& Kahn 1997), since particle motion is described by trajectories. Now a stream of particles is represented no longer by a single classical trajectory, but by a bundle of stochastic trajectories, changing randomly with time, and having a tree-like structure, although reaching the same values of $\gamma$; and, unlike in the classical regime, the energy no longer has a smooth growth but shows chaotic behavior. Thus, pulsar magnetospheres, when radiation starts to influence particle motion, become random media with fractal structure.

Pulsars are compact stars requiring geometrodynamics where q-classical regimes are not Markovian. This only happens in the context of Minkowski 4space. But the 4-space curvature at the CP SLC is a perturbation, and therefore the q-classical regime is quasi-Markovian there (Andrade 1994).

\section{The Generalized Quantum Connection: The Ginzburg Effect}

Q-classical regimes start to be important when $\gamma B \sim 4 \times 10^{9} \mathrm{~T}$ and vacuum polarization starts to play an important role. Not only does the vacuum become unstable (Fradkin, Gitman, \& Shvartzman 1991), it also becomes an inhomogeneous and anisotropic medium (Ginzburg 1989). This means that a stream of highly energetic particles might activate the unstable vacuum, triggering the production of charged particles from vacuum; but, due to quantum vacuum properties, new forms of radiation might exist, such as transition radiation, transition scattering, and bremsstrahlung as defined by Ginzburg \& Tsytovitch (1990), which add up to the standard curvature radiation effects.

The vacuum must have a tendency to become highly dense when the particles' $\gamma$ grows. Then $\gamma<10^{3}$ for the stellar surface; and $\gamma<10^{9}$ for the CP SLC.

Acknowledgments. Most of the present work was produced in close cooperation with the late Professor F. D. Kahn.

We thank the Luso-American Development Foundation, Portugal, for financial support of our presence in this symposium.

\section{References}

Andrade, Z. 1994, M.S. Thesis, Instituto Superior Técnico, Lisboa, Portugal da Costa, A. A. 1983, MNRAS, 204, 1125

da Costa, A. A. \& Kahn F. D. 1982, MNRAS, 199, 211

da Costa, A. A. \& Kahn F. D. 1997, MNRAS, 284, 1

Fradkin, E. S., Gitman, D. M., \& Shvartzman, S. M., 1991, Quantum Electrodynamics with Unstable Vacuum (Berlin: Springer)

Ginzburg, V. I. 1989, Applications of Electrodynamics in Theoretical Physics and Astrophysics (London: Gordon \& Breach)

Ginzburg, V. I., Tsytovitch, V. N. 1990, Transition Radiation and Transition Scattering (London: Adam Hilger) 